\title{
résistance en compression d'un lignite terrigène
}

\author{
par \\ A. G. Anagnostopoulos, \\ Privat-docent, Chaire des Fondations, \\ Université Technique Nationale d'Athènes (U.T.N.A.), Grèce
}

\section{Introduction}

En Grèce, le bassin de Mégalopolis, situé dans le Péloponnèse, est connu pour ses gisements étendus de lignite, qui constituent une des sources les plus importantes de combustibles solides du pays. Leur exploitation se fait "à ciel ouvert", procédure qui conduit à la formation de talus de hauteur importante, consistant principalement en couches successives de lignite et de dépôts terrigènes. D'autre part, un nombre considérable d'ouvrages de Génie Civil supportant des charges importantes ont été fondés sur le profil géotechnique évoqué ci-dessus. Dans le cas où le lignite se trouve en abondance dans le sous-sol (cas de Mégalopolis), ses caractéristiques physiques et mécaniques influencent très nettement le comportement du terrain. Toutefois, les principes de base du comportement du lignite ne sont pas toujours clairement établis. Une recherche a été réalisée à I'U.T.N.A. (Anagnostopoulos A., 1980) visant l'étude des facteurs qui influencent la résistance et la déformabilité du lignite de Mégalopolis.

Le présent article expose les résultats de cette recherche en se référant aux caractéristiques de résistance en compression triaxiale (CIU), l'anisotropie et l'effet du taux de déformation ayant été étudiés dans une série d'essais de compression simple.

\section{Aperçu de la géologie du bassin}

Le bassin de Mégalopolis, de $252 \mathrm{~km}^{2}$ de surface, en forme de quadrilatère, se trouve à peu près au centre du Péloponnèse. II constitue un graben interne et son remplissage a commencé au Pliocène Supérieur. Dans ce bassin, des sédiments lacustres et torrentiels subaériens se sont déposés successivement pendant le Pliocène Supérieur et le Pleistocène Inférieur, tandis que d'autres sédiments torrentiels et continentaux se sont également déposés pendant le Pleistocène Supérieur et l'Holocène. Ces dépôts sédimentaires sont composés de marnes argileuses, de sables, de conglomérats et de microconglomérats polygéniques, plus ou moins indurés. Ainsi, au début de l'époque Quaternaire, grâce à l'accumulation phytogène aquatique, s'est formé le lignite terrigène de Mégalopolis, tendre et riche en eau.

La géologie et la géomorphologie de ce bassin ont fait l'objet de plusieurs études (Phillipson A., 1891, Marinos et al, 1959, Lutting et Marinos, 1962, etc.), ces auteurs traitant de la géologie de ce bassin et des matériaux qui $y$ ont été déposés. Le gisement lignitifère de Mégalopolis est composé de plusieurs couches de lignite qui sont séparées par plusieurs couches terrigènes d'épaisseur variable. Ces dernières comprennent des argiles, des pélites ainsi que des argiles pélitiques. On y remarque aussi le passage progressif des lignites vers des argiles sapropéliques ou des argiles simples.

Le point remarquable de ce bassin lignitifère, c'est que les couches de lignite sont horizontales sans pendage ou rejet de faille visibles (Marinos et al, 1959). La figure 1 présente une coupe schématique $(\mathrm{N}-\mathrm{S})$ des couches lignitifères du bassin.

\section{Caractéristiques physiques du lignite}

Le gisement de lignite de Mégalopolis est un matériau terrigène humide et $*$ mou $n$, dont la structure est spongieuse. II contient des minéraux argileux qui se distribuent dans sa masse d'une manière plutôt uniforme. Sa couleur normale est le brun foncé virant au noir lorsqu'il est exposé à l'atmosphère (oxydation). Au point de vue géotechnique, il se présente comme un matériau saturé de sorte que, lorsqu'il est immergé dans l'eau, il n'en absorbe pratiquemment que très peu ou pas du tout. Dans son état naturel, dégagé de l'humidité et de la cendre, la consistance chimique de ce lignite est approximativement la suivante : volatiles $62 \%$, charbon fixé $38 \%$. Sa valeur calorifique est relativement basse (de l'ordre de $100 \mathrm{Kcal} / \mathrm{kg}$ à l'état 


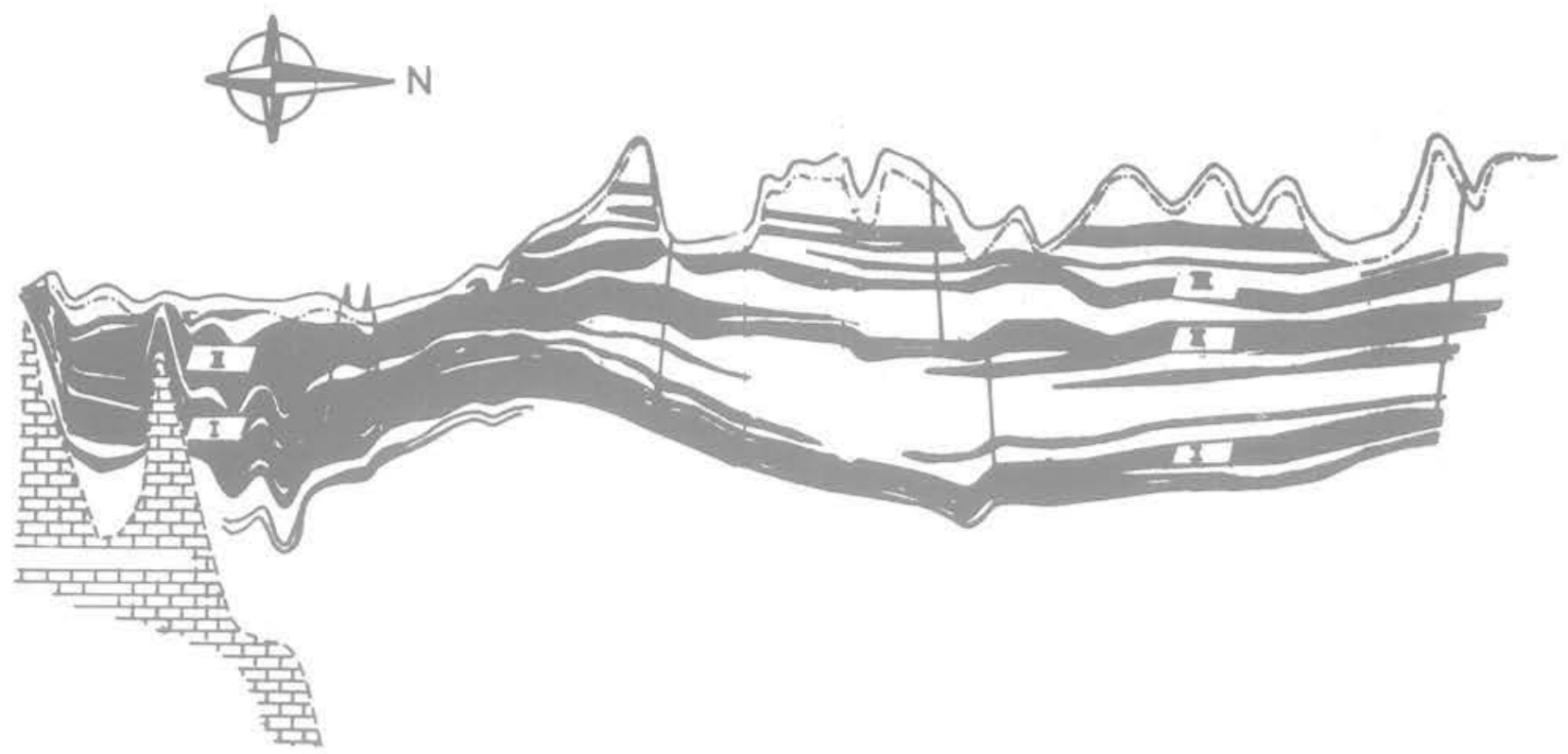

Fig. 1 Coupe schématique longitudinale (N-S) des gisements lignitifères du bassin de Mégalopolis (H. Georgen, 1968)

sec) (Marinos et al, 1959). Vu à son humidité naturelle élevée, il se classe dans la gamme des lignites les plus "pauvresn de l'Europe.

La teneur en eau du lignite de Mégalopolis varie entre 120 et $180 \%$ (poids constant à $60^{\circ} \mathrm{C}$ ); le poids volumique humide est d'environ $11,5 \mathrm{kN} / \mathrm{m}^{3}$; le poids volumique du squelette solide (matrice ligniteuse) varie entre 16,5 et $17,2 \mathrm{kN} / \mathrm{m}^{3}$. Cette variation des caractéristiques physiques du lignite peut être attribuée aussi bien à sa nature qu'à la présence de minéraux argileux dans sa masse.

Les caractéristiques physiques des dépôts terrigènes déposés parmi les couches de lignite prennent les valeurs suivantes : teneur en eau, près de $30 \%$; poids volumique humide, entre 17,5 et $19,5 \mathrm{kN} / \mathrm{m}^{3}$; poids volumique des grains, environ 25 à $27 \mathrm{kN} / \mathrm{m}^{3}$. Suivant la classification de Casagrande, ces matériaux se classifient généralement comme ML et OL.

oủ $\mathrm{fO}-$ Lp $(3<\mathrm{MO} \leqslant 10 \%$, sol limoneux $)$

$$
\text { Lp Op } \longleftarrow \text { LPC }
$$

\section{Expérimentation}

La préparation des échantillons de lignite pour l'expérimentation a été réalisée de la façon suivante : des blocs cubiques $(0,4 \times 0,4 \times 0,4 \mathrm{~m})$, prélevés dans les talus de la mine et emballés dans des sacs doubles en polyéthylène, ont été coupés en morceaux à l'aide d'un canif et façonnés par la suite à la main sous forme de cylindres de $0,035 \mathrm{~m}$ de diamètre et $0,07 \mathrm{~m}$ de hauteur. La microstratification du lignite dans les éprouvettes était horizontale, suivant la stratigraphie générale du bassin. Afin d'éviter toute oxydation, les éprouvettes cylindriques de lignite ont été conservées dans un bain d'eau vidé de son air. Cette dernière procédure suit les principes donnés par Trollope et al, (1965), principes utilisés dans l'exploitation des lignites d'Australie.
Les essais triaxiaux non-drainés ont été réalisés à vitesse constante $\left(\mathrm{v}=7,5 \cdot 10^{-7} \mathrm{~m} / \mathrm{s}\right)$, les échantillons ayant subi une consolidation isotrope. La saturation de ces derniers a été assurée par contre-pression. Le mode opératoire suit les principes donnés par Bishop et Henkel (1962), tandis que le critère de rupture adopté a été celui de la contrainte déviatorique maximale.

Les résultats de la recherche concernant la déformabilité du lignite de Mégalopolis (Anagnostopoulos A. 1980 ) ont montré que la contrainte de préconsolidation des couches superficielles est constante, de l'ordre de $950 \mathrm{kPa}$, et identique pour le lignite et les dépôts terrigènes, compte tenu aussi du fait que les paramètres de résistance ont été étudiés dans une gamme de pressions latérales allant jusqu'à $2800 \mathrm{kPa}$.

Les essais de compression simple, utilisés pour l'étude de l'influence du taux de déformation sur la résistance, ont été réalisés à vitesse constante, variant dans les divers essais entre $7,5 \cdot 10^{-7}$ et $6,33 \cdot 10^{-5} \mathrm{~m} / \mathrm{s}$, les échantillons étant à stratification horizontale. Lors de l'étude de l'anisotropie, la vitesse $\varepsilon_{1}$ a été fixée à $8,33 \cdot 10^{-6} \mathrm{~m} / \mathrm{s}$ et la compression a été appliquée suivant trois directions : orthogonale, parallèle et sous un angle de $45^{\circ}$ par rapport à la stratigraphie. Comme pour les essais triaxiaux, tous les échantillons testés en compression simple ont été saturés par contrepression.

\section{Caractéristiques de résistance}

\subsection{Lois contrainte-déformation}

Les résultats des essais ont mis en évidence le fait que, pour un même niveau de déformation, la contrainte déviatorique augmente avec la contrainte latérale (pression de consolidation); il en est de même pour la déformation à la rupture. En termes de contrainte 


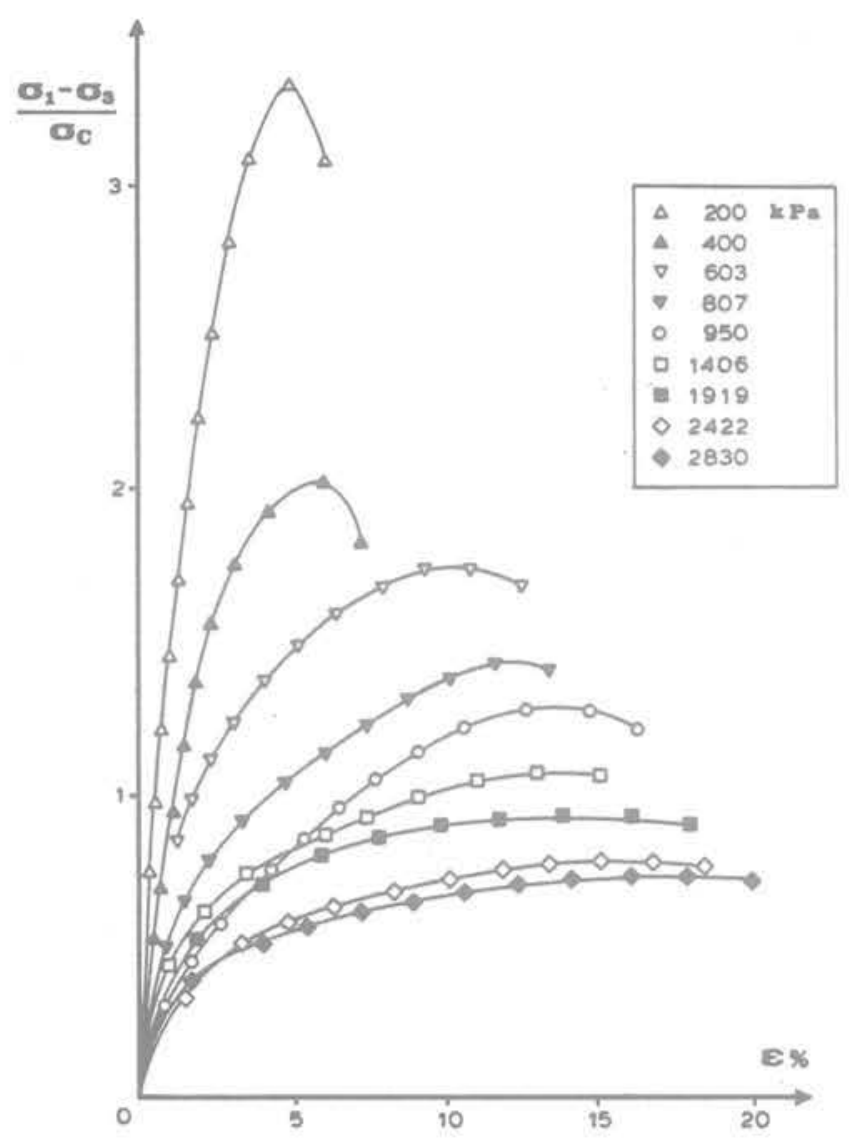

Fig. 2 Variation de la contrainte déviatorique normalisée, $\sigma_{d n}$, avec la déformation axiale, $\epsilon_{1}$

déviatorique normalisée $\sigma_{\mathrm{dn}}$ et de déformation axiale $\varepsilon_{\text {, }}$ les résultats indiquent (fig. 2) que le concept de Ladd (1964) concernant le comportement normalisé des argiles est également valable pour le lignite étudié. De cette même figure il ressort que, pour une faible pression de consolidation, la pente à l'origine de ces courbes est assez grande, beaucoup plus que pour une pression de consolidation élevée. A la rupture, les valeurs maximales de $\sigma_{\mathrm{dn}}$ augmentent avec la pression de consolidation. Dans le cas où cette dernière devient égale ou supérieure à la pression de préconsolidation, on observe une nette tendance des résultats expéri- mentaux à se regrouper autour d'une relation unique entre $\sigma_{c n}$ et $\varepsilon_{1}$. Comme prévu, il existe une dispersion des résultats des essais, dispersion qu'on peut également noter sur des argiles surconsolidées (Ladd et Foott, 1974); dans notre cas, ce phénomène est plutôt attribué à l'hétérogénéité du matériau.

La figure 3, représentant la variation de la contrainte déviatorique normalisée à la rupture, $\sigma_{\text {dnf, }}$ avec la pression de consolidation met en relief l'effet de la surconsolidation sur la résistance du lignite. Ce même phénomène apparaît également sur la figure 4, où $\sigma_{\text {dnt }}$ est lié au rapport de surconsolidation (OCR). II faut s'attendre à ce que, plus cette relation est linéaire, plus l'enveloppe de Mohr soit non-linéaire.

La pression interstitielle, u, qui se développe au cours de l'essai triaxial CIU, ne s'accroît que très peu au début de l'essai,-avec toutefois un taux d'accroissement accéléré par la suite.

Ce phénomène est dû probablement à la vitesse de l'essai qui ne permet pas l'égalisation des pressions interstitielles à l'intérieur de l'échantillon, pendant les premières phases de l'essai. Au pic (rupture), la pression interstitielle est approximativement de 55 à $70 \%$ de la contrainte latérale; ce dernier phénomène montre que la contrainte effective latérale est assez basse. Après le pic, la pression interstitielle augmente continuellement dans les éprouvettes normalement consolidées, tandis qu'elle diminue - très peu d'ailleurs - dans les éprouvettes surconsolidées. Le diagramme de la figure 5 , qui exprime la relation entre la pression interstitielle normalisée et la déformation axiale, vérifie la validité du concept de Ladd (1964) dans le cas du lignite étudié. C'est ainsi que les courbes se référant aux éprouvettes normalement consolidées ont tendance à se regrouper, tandis que celles des éprouvettes surconsolidées s'en écartent. Les essais triaxiaux exécutés ont permis la détermination du paramètre $A$. de Skempton. Pour un lignite normalement consolidé, la valeur $A_{t}$ de ce paramètre à la rupture est du méme ordre de grandeur que celui des argiles légèrement surconsolidées. Pour un lignite surconsolidé, $A_{f}$ est toujours positif, en tout cas supérieur à 0,2 , même dans le cas où le rapport de surconsolidation atteint la valeur de 5 . La variation de $A_{1}$ avec la pression de consolidation $\sigma_{c}$ est illustrée dans la figure 6 . D'après cette figure, il est clair que $A_{\text {, }}$ s'accroît avec $\sigma_{c}$ pour les éprouvettes normalement

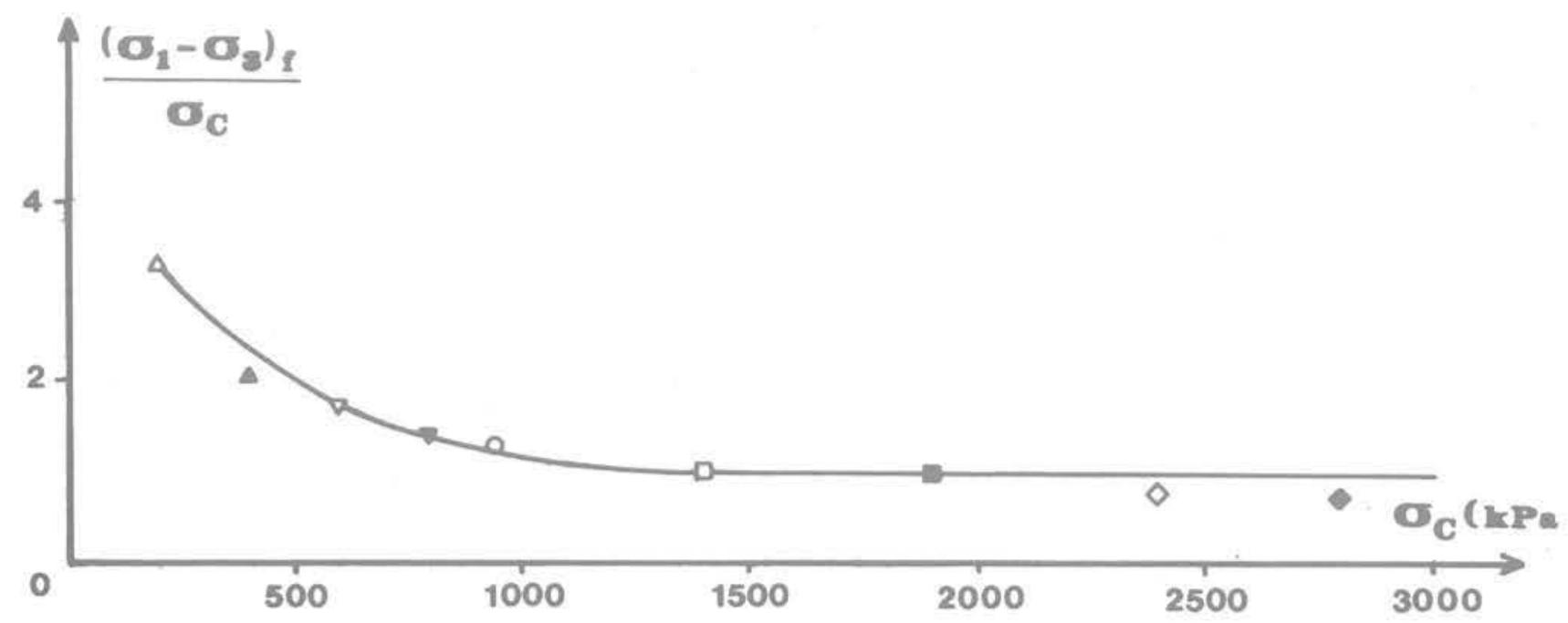

Fig. 3 Variation de la contrainte déviatorique normalisée à la rupture, $\sigma_{d n f}$, avec la pression latérale, $\sigma_{C}$ 


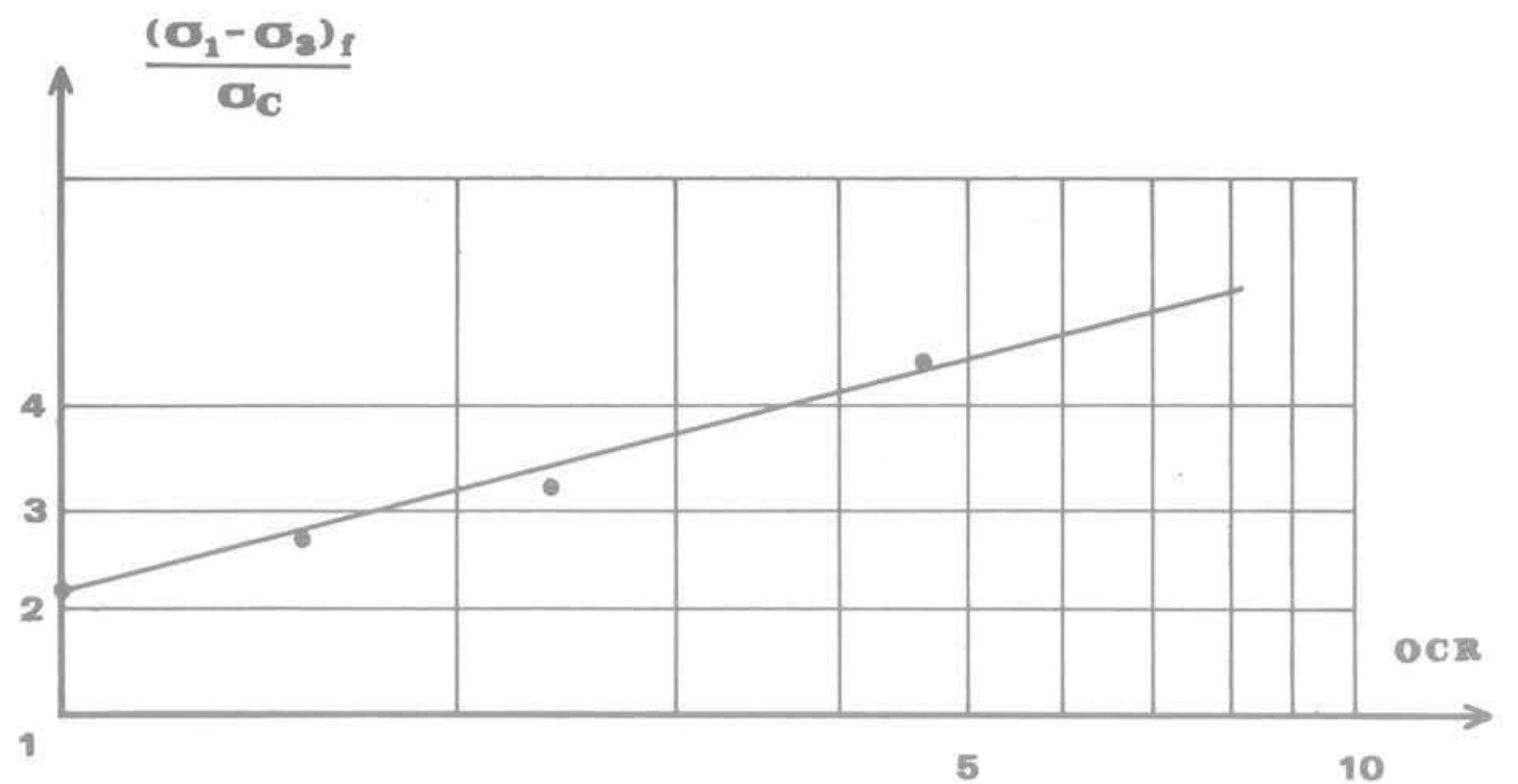

Fig. 4 Relation entre la contrainte déviatorique normalisée à la rupture, $\sigma_{d n f}$ et le rapport de surconsolidation

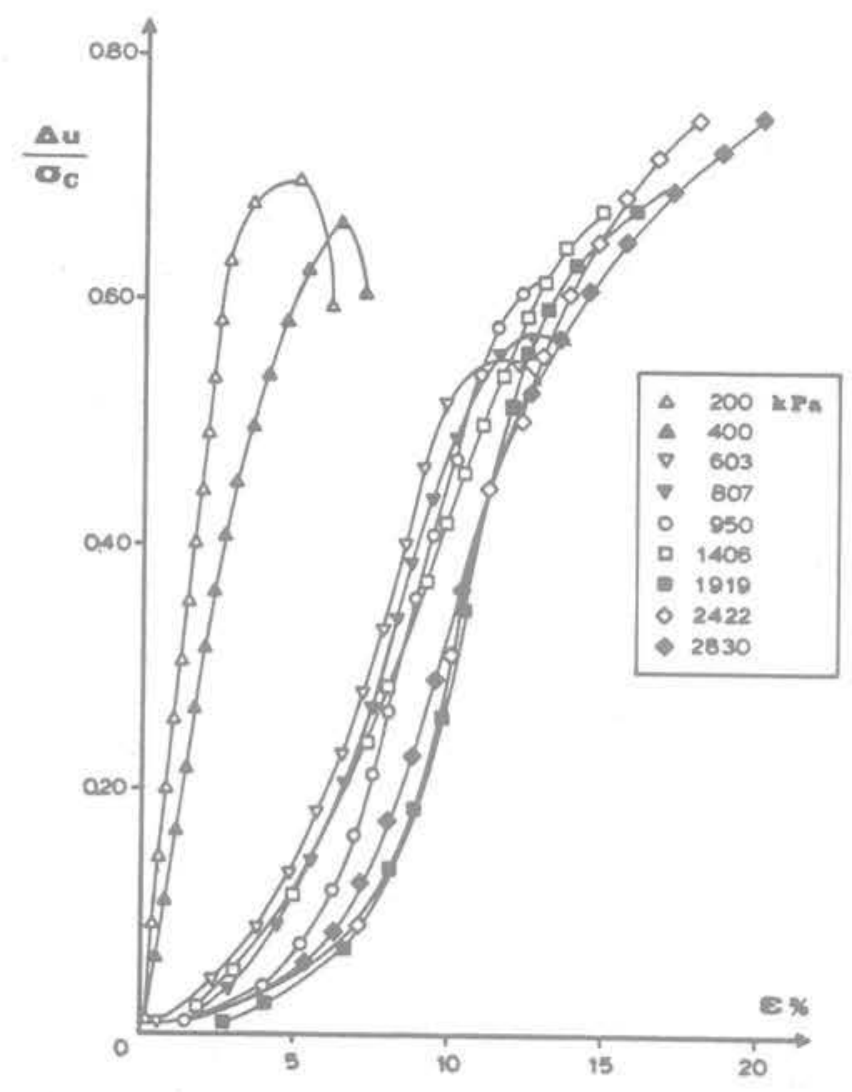

Fig. 5 Variation de la pression interstitielle normalisée, $u_{n}$, avec la déformation axiale, $\epsilon_{1}$

consolidées; ce phénomène devrait être associé au changement de la structure du lignite qui survient lorsque la contrainte latérale augmente. Pour les pressions latérales élevées $\left(\sigma_{c}=2800 \mathrm{kPa}\right), A_{t}$ s'approche de 1; en d'autres termes, le lignite manifeste un comportement semblable à celui d'une argile pure. L'influence de $\sigma_{c}$ sur $A_{t}$, comme indiqué ci-dessus, se reflète dans l'allure de l'enveloppe de Mohr dans un diagramme en contraintes totales.

\subsection{Paramètres de résistance}

Les chemins de contraintes effectives correspondant aux éprouvettes de lignite normalement consolidé ou légèrement surconsolidé ont l'allure de ceux qu'on retrouve pour des argiles normalement consolidées. Dans le cas où le rapport de surconsolidation OCR est élevé (OCR $>4)$, cette allure ressemble plutôt à celle des argiles surconsolidées (fig. 7). Ce comportement peut être attribué au mécanisme de génération des pressions interstitielles.

Les observations faites sur les éprouvettes de lignite testées ont montré que la rupture est survenue sur chaque échantillon suivant un "plan de rupture", justifiant ainsi le critère de rupture adopté. Par rapport à l'horizontale, les plans de rupture observés ont une inclinaison variant entre $58^{\circ}$ et $62^{\circ}$. Ici, on doit ajouter que dans certains cas la rupture s'est manifestée suivant deux plans conjugués, rupture semblable à celle qui apparaît normalement dans le cas des matériaux homogènes et fragiles. Toutefois, le lignite étant non-homogène, toute tentative de similitude avec un matériau homogène-fragile paraít impossible.

Les paramètres de résistance en contraintes effectives et en contraintes totales ont été déterminés à l'aide de l'enveloppe conventionnelle de Mohr et de l'enveloppe qui figure sur le diagramme $p-q$ (chemin des contraintes). En termes des contraintes totales (fig. 8), on peut observer l'allure courbée mentionnée cidessus, principalement lorsque la pression latérale est élevée. Ce phénomène peut être attribué à la présence de hautes pressions interstitielles qui se développent à la rupture. Sur la partie de l'enveloppe qui correspond à la surconsolidation, les paramètres de résistance atteignent les valeurs $c=200 \mathrm{kPa}$ et $\phi=16^{\circ}$. Une forme analogue d'enveloppe a été également observée par Brown E. (1963) sur les lignites terrigènes Morwell d'Australie.

Les deux figures 7 et 9 (contraintes effectives) montrent bien que l'enveloppe de Mohr peut être remplacée avec succès par deux droites, la première correspondant à l'état de surconsolidation et la deuxième à l'état de consolidation normale, les paramètres de résistance ayant respectivement les valeurs $c^{\prime}=180 \mathrm{kPa}, \phi^{\prime}=26,7^{\circ}$ et $c^{\prime}=150 \mathrm{kPa}, \phi^{\prime}=$ $28,5^{\circ}$. Il est clair que, dans ce deuxième cas, le lignite 


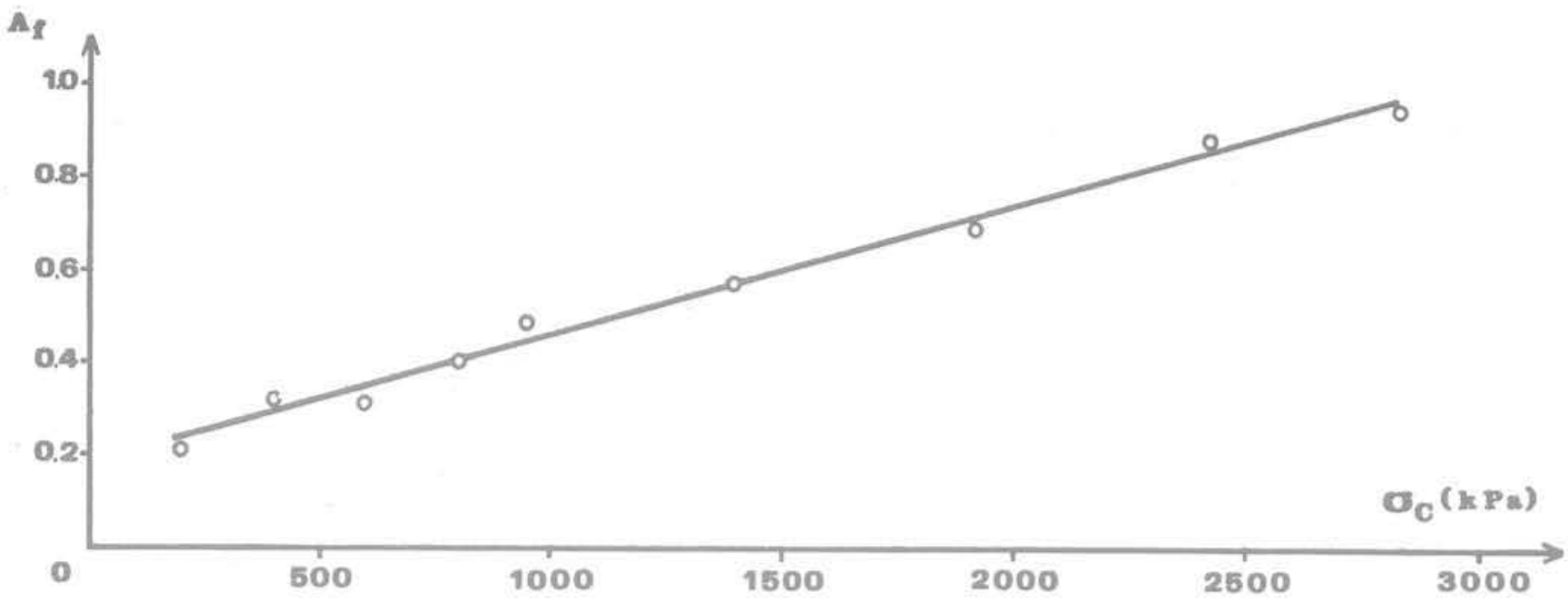

Fig. 6 Variation du paramètre $A_{f}$ avec la pression de consolidation, $\sigma_{c}$

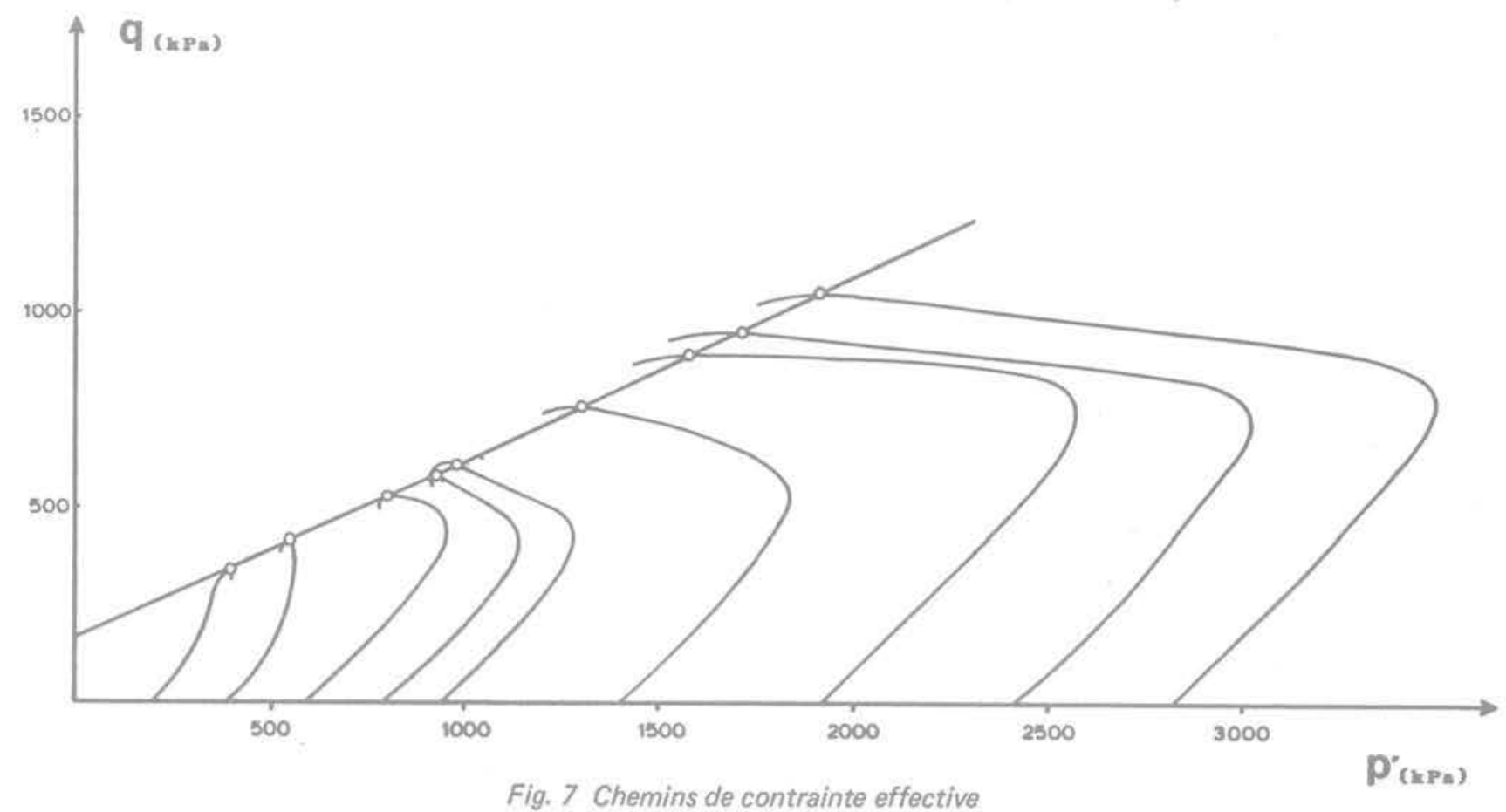

Fig. 7 Chemins de contrainte effective

dispose d'une cohésion, contrairement aux argiles. $\mathrm{Ce}$ phénomène est également signalé par Trollope et al. (1965) sur des lignites d'Australie et ces auteurs l'ont attribué aux liaisons chimiques qui existent entre les divers éléments du squelette des lignites.

\subsection{Taux de déformation}

La figure 10 montre l'accroissement de la résistance (pic) suivant le taux de déformation sur des échantillons ayant une stratigraphie horizontale. Dans un diagramme semi-logarithmique, la loi est linéaire et la résistance non-drainée s'accroît de près de $38 \%$ pour une augmentation du taux de déformation d'un facteur 100.

Ce comportement se manifeste généralement chez les argiles et divers auteurs ont admis une loi linéaire dans un diagramme semi-logarithmique. Toutefois, par rapport aux argiles surconsolidées, le taux d'accroissement de la résistance du lignite est relativement important.

\subsection{Anisotropie}

La figure 11 montre la variation de la résistance non-drainée $c_{u}$ avec la direction du chargement. Les valeurs adoptées de $c_{u}$ pour chaque direction du chargement représentant la moyenne de six séries d'essais où l'écart se situait entre 3,9 et $6,5 \%$. Cette variation de $c_{u}$ se présente de la même façon que celle apparue sur les argiles stratifiées (Yong et Workentin. 1975); elle approche sensiblement la loi bien connue de variation elliptique de Casagrande et Carrillo (1944). Le rapport des demi-axes de l'ellipse $\left(c_{u v} / c_{u h}\right)$ est proche de 1,5 . 


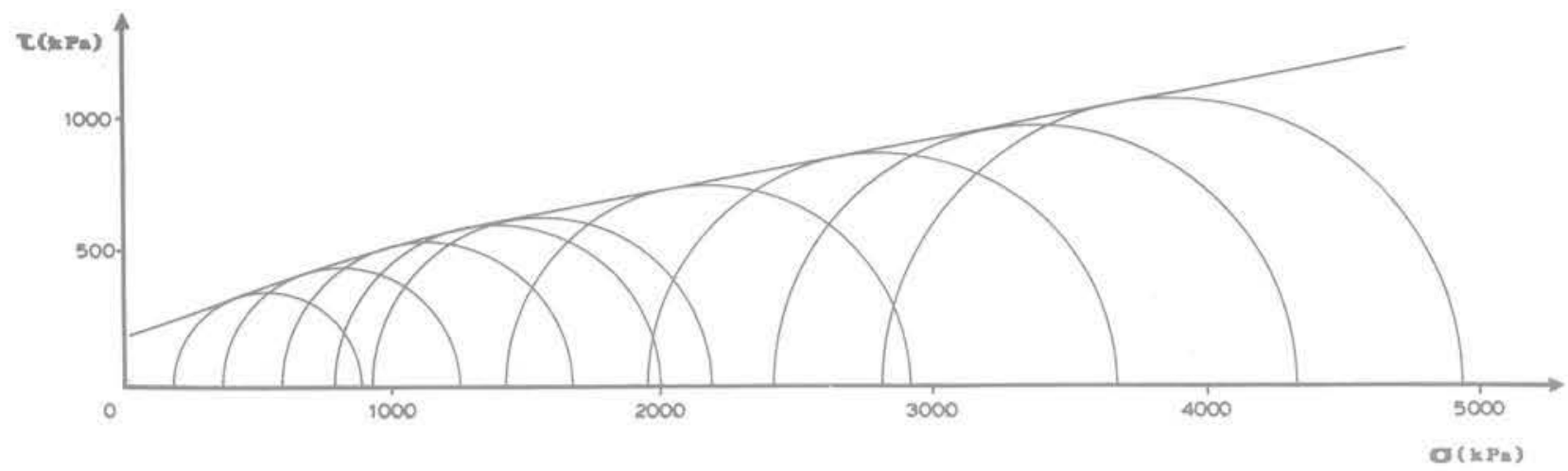

Fig. 8 Enveloppe de rupture (Mohr), contraintes totales

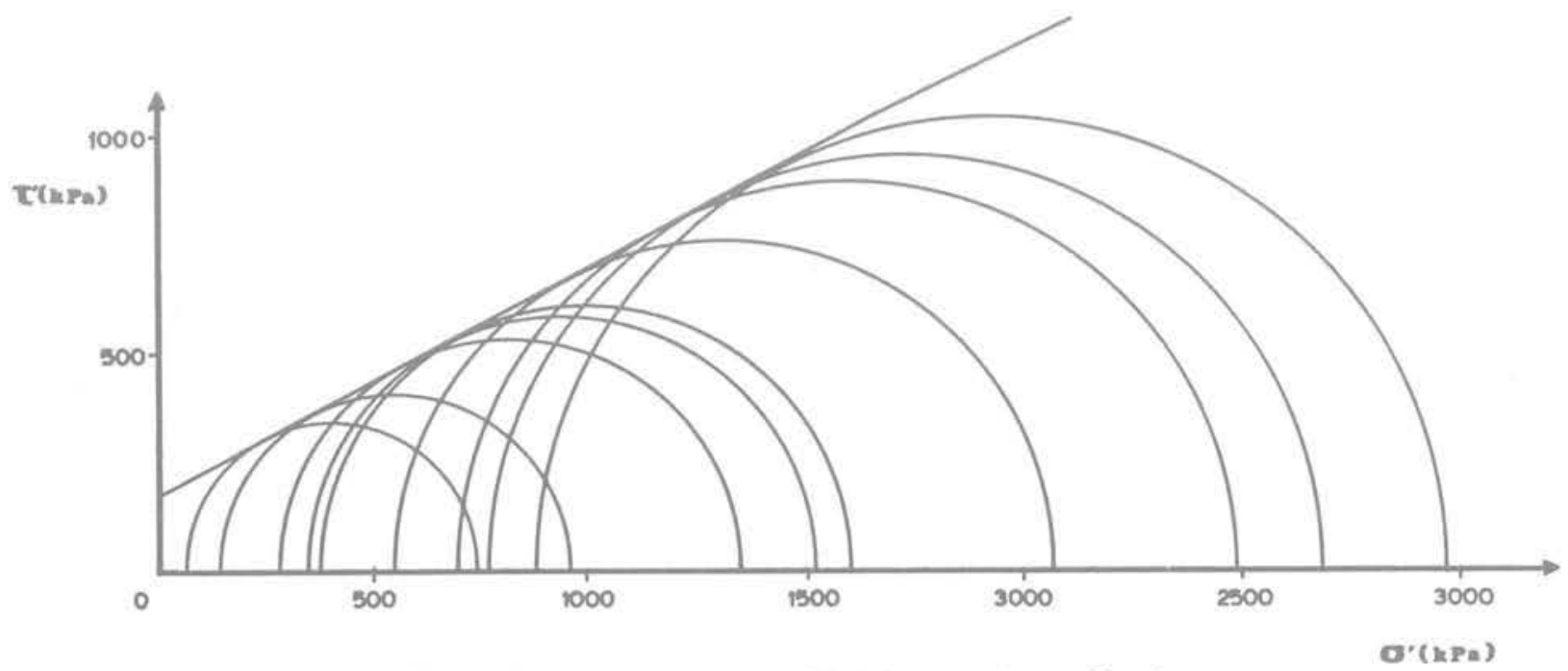

Fig. 9 Enveloppe de rupture (Mohr), contraintes effectives

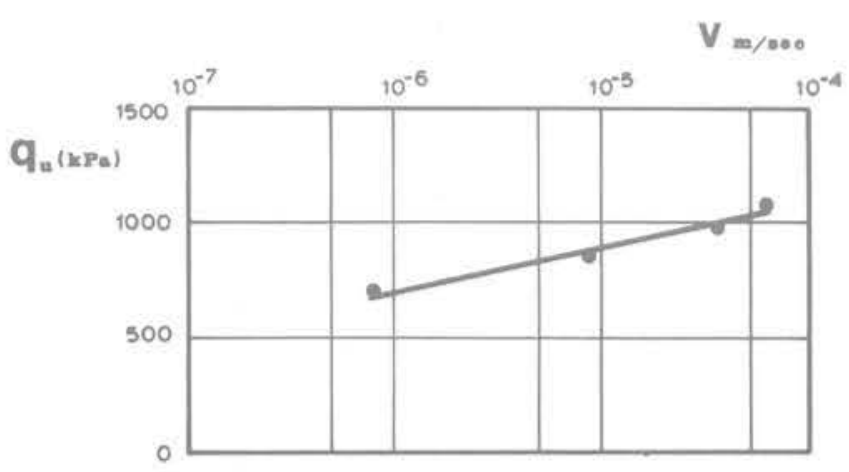

Fig. 10 Influence de la vitesse de chargement sur la résistance non-drainée, $c_{u}$

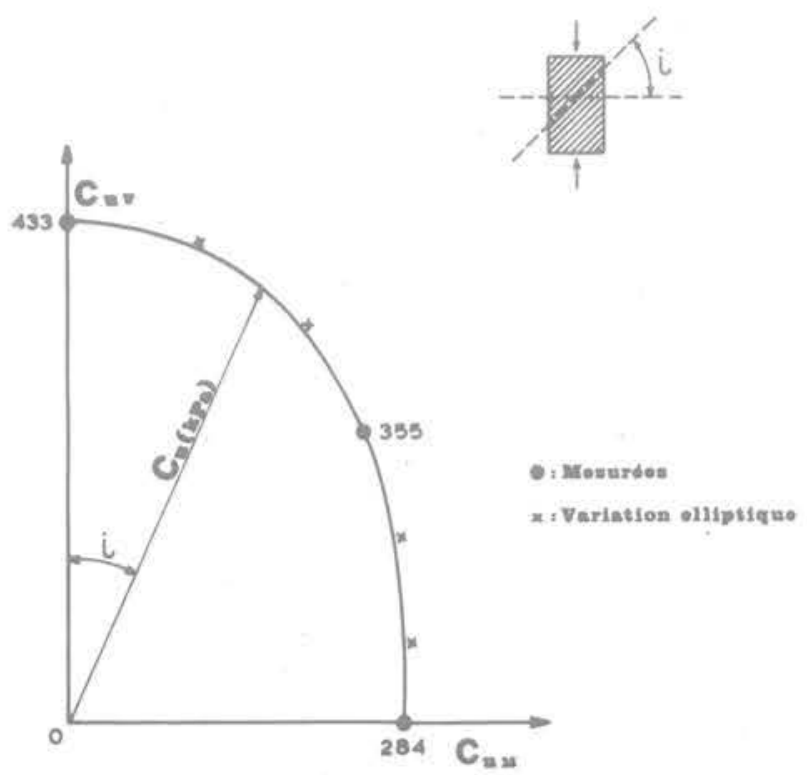

Fig. 11 Variation de la résistance non-drainée avec la direction de la contrainte appliquée 


\section{Conclusions}

La résistancè en compression du lignite de Mégalopolis, en Grèce, a été étudiée à l'aide d'une série d'essais triaxiaux CIU sur éprouvettes ayant une stratification horizontale, suivant celle des gisements rencontrés in situ. Le matériau étudié est surconsolidé et, dans un diagramme de contraintes effectives, on peut distinguer nettement deux segments rectilignes de l'enveloppe de rupture (Mohr). L'allure de cette dernière est analogue à celle rencontrée sur les argiles surconsolidées, à la seule différence que la partie correspondant aux éprouvettes normalement consolidées est douée de cohésion. Ce phénomène est attribué à la présence de liaisons chimiques entre les feuillets du lignite. La pression interstitielle qui se développe au cours des essais évoqués ci-dessus est importante, allant jusqu'à 55 à $70 \%$ de la pression latérale au moment de la rupture; elle est cependant toujours positive, même en cas d'éprouvettes fortement surconsolidées (OCR $>4$ ). En termes de contraintes totales, le segment de l'enveloppe de rupture correspondant à la surconsolidation est nettement linéaire, tandis qu'au-delà, on peut observer la présence d'une partie courbe de l'enveloppe ayant sa concavité dirigée vers le bas; ceci est dû à l'accroissement avec la pression latérale du paramètre $A$ de Skempton. Dans cette même partie, ce

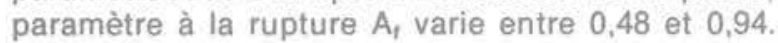

Les chemins de contraintes effectives du lignite étudié ressemblent à ceux des argiles normalement consolidées sauf pour un OCR $>4$ où la ressemblance va plutôt aux argiles surconsolidées. Les diagrammes normalisés $\sigma_{d n}-\varepsilon_{1}$ et $u_{n}-\varepsilon_{1}$ prouvent que le principe de Ladd (1964) concernant les argiles est également valable pour le lignite étudié, et ceci est une précision satisfaisante. On peut dire ainsi que le comportement du lignite de Mégalopolis est analogue à celui des argiles, surtout lorsque le matériau est normalement consolidé.

Lors de deux séries d'essais de compression simple ayant comme but l'étude de l'influence sur la résistance non-drainée, d'une part de la vitesse de chargement et, d'autre part, de l'anisotropie du matériau suivant trois axes de chargement, il a été observé que la résistance augmente linéairement avec le logarithme de la vitesse de chargement, tandis que la variation de la résistance non-drainée est elliptique, suivant l'orientation de l'axe de chargement. Le rapport de deux demi-axes de l'ellipse $\left(c_{u v} / c_{u h}\right)$ est proche de 1,5

La recherche effectuée aboutit à cette conclusion générale que le comportement mécanique de divers matériaux terreux non usuels (par exemple le lignite) peut être étudié avec les méthodes courantes de la Mécanique des Sols traditionnelle.

\section{Références bibliographiques}

A.G. Anagnostopoulos (1980), "Comportement géotechnique du lignite de Mégalopolis " (en Grec). Thèse présentée à I'Université Technique Nationale d'Athènes, Grèce 1980, pour l'obtention du titre de Docent (U.T.N.A.)

A. Bishop, D. Henkel (1962), "Consolidated-Undrained Tests". The measurement of soil properties in the triaxial test, $2^{\circ}$ édition Eduard Arnold (Publishers) Ltd., London, 1962, pages 106-121.
E.T. Brown (1963), "The Engineering Behaviour of Morwell Brown Coal». Thèse de Master présentée à I'Université de Melbourne, Australie, 1963, page 173.

A. Casagrande, N. Carillo (1944), "Shear failure of anisotropic materials". Proceedings of the Boston Society of Civil Engineers, 1944, vol. 31, pages 74-87.

H. Georgen (1968), "Rahmenplanung für den übertägigen Abbau der heizwertarmen Braunkohle des Reviers Megalopolis (Griechenland) ». Förden und Heben-Heft 11, 1968, s. 1-8.

C.C. Ladd (1964), "Stress-strain characteristics and basic strength principles". Research Report R64-17Dept. of Civil Engineering M.I.T., Cambridge, Mass., 1964

C.C. Ladd, R. Foott (1974), « New design procedure for stability of soft clays". Journal of the Geotechnical Engineering Division - Proceedings of the A.S.C.E. vol. 100, n GT7, juillet 1974, pages 763-786.

G. Lütting, G. Marinos (1962), «Zur Geologie der neuen Griechischen Braunkohlen Lagerstatte von Megalopolis ». Braunkohle Wärme und Energie-Heft 14.

Deutschland, 1962, s. 222-231.

G. Marinos, J.Anastopoulos, N. Papanicolaou (1959), "Le Lignite du Bassin de Mégalopolis" (en Grec). Recherches en Géologie et Géophysique, vol. $15, n^{\circ} 3$. Institut de Géologie et de Recherche Souterraine, Athènes, Grèce, 1959, pages 1-51.

A. Philippson (1891), "Peloponnesische Bergfahrten ". Zeitschrift Deutscher-Östereichische Alpenvereins. Deutschland, vol. 22, 1891 , s. 282-315.

D.H. Trollope, K.J. Rosengren, E.T. Brown (1965), "The Mechanics of Brown Coaln. Géotechnique, vol. $15, n^{\circ} 4,1965$, pages $363-386$.

R.N. Yong, B.P. Warkentin (1975), "Anisotropic effects ". Soil Properties and Behaviour. Elsevier Scientific Publishing Company, Amsterdam, Oxford, New York, 1975, pages 356-359.

\section{Notations}

$\varepsilon_{1}=$ déformation axiale

$\sigma \quad=$ contrainte normale.

$\sigma_{c} \quad=$ pression de consolidation pression latérale.

$\sigma_{\mathrm{dn}}=$ contrainte déviatorique normalisée. $\frac{\left(\sigma_{1}-\sigma_{3}\right)}{\sigma_{0}}$.

$\sigma_{\mathrm{dnt}}=$ contrainte déviatorique normalisée à la rupture, $\frac{\left(\sigma_{1}-\sigma_{3}\right)_{t}}{\sigma_{c}}$.

$\mathrm{u} \quad=$ pression interstitielle.

$\mathrm{u}_{n} \quad=$ pression interstitielle normalisée, $\mathrm{u}_{\mathrm{n}}=\mathrm{u} / \sigma_{\mathrm{c}}$.

$\mathrm{CIU}=$ essai triaxial non-drainée, consolidé isotropiquement.

$\mathrm{c}_{\mathrm{u}} \quad=$ résistance non-drainée.

$\mathrm{c}_{\mathrm{uv}}=$ résistance non-drainée pour un chargement orthogonal à la stratigraphie.

$\mathrm{c}_{\mathrm{uh}}=$ résistance non-drainée pour un chargement parallèle à la stratigraphie.

v $\quad=$ vitesse de chargement .

OCR $=$ rapport de surconsolidation

$\tau=$ contrainte de cisaillement. 
